\title{
News from Lattice QCD
}

\author{
Peter Weisz \\ Max-Planck-Institut für Physik \\ Föhringer Ring 6, 80805 Munich - Germany
}

\begin{abstract}
In this short talk I will present some recent developments from lattice QCD. The presentation is prepared for non-experts with emphasis on general information which will hopefully act as a guide on how to assess phenomenological results presented from various lattice simulations.
\end{abstract}

\section{Introduction}

Quantum chromodynamics is the commonly accepted candidate theory of the strong interactions. Its action, describing the interactions of quarks and gluons:

$$
S=\int \mathrm{d}^{4} x\left\{\frac{1}{2} \operatorname{tr} F_{\mu \nu} F^{\mu \nu}+\sum_{f} \bar{q}_{f}\left[i \gamma D+m_{f}\right] q_{f}\right\},
$$

is rather esthetic, apart from the quark mass terms. Various high energy processes can be reliably computed using renormalized perturbation theory because of the property of asymptotic freedom. The results thereby obtained are in all cases certainly consistent with experiment. However we should also compute low energy properties of the bound states (hadrons) from first principles. If such QCD computations agreed with experiment to a satisfactorily good precision, then we could really accept QCD as the theory of strong interactions. It is often underestimated what a great achievement this would be!

For example to obtain the pion mass we would like to compute the (Euclidean) two-point function of local fields with pion quantum numbers for large time separations:

$$
\langle\pi(t) \pi(0)\rangle \propto \int[\mathrm{d} A \mathrm{~d} \bar{q} \mathrm{~d} q] \mathrm{e}^{-S[A, \bar{q}, q]} \pi(t) \pi(0) \sim \mathrm{e}^{-m_{\pi} t}
$$

The expression above is however formal because we don't know how to define the measure in the continuum, outside the framework of perturbation theory. The only known way how to approach this difficulty and to obtain a non-perturbative definition of the theory is to employ lattice regularization where the continuum space-time is replaced by a (hyper-cubic) lattice. Euclidean invariance is broken but gauge invariance is maintained. QCD should however be obtained in the limit of taking the lattice spacing $a$ to zero.

In such a short review it is impossible to present many details of the theoretical framework. Here we only recall that instead of the local gauge field $A_{\mu}$ we now deal with variables $U_{\mu}(x) \in \mathrm{SU}(3)$ associated with the links joining a point $x$ to a neighboring point $x+a \hat{\mu}$ on the lattice, which correspond to parallel transporters:

$$
U_{\mu}(x) \sim P \exp \int_{0}^{1} \mathrm{~d} t A_{\mu}(x+\operatorname{ta\hat {\mu }})
$$


Quark fields $q(x)$ and antiquark fields $\bar{q}(x)$ are defined at points $x$ on the lattice $\Lambda$. The formal measure in (2) is then replaced by

$$
\int[\mathrm{d} A \mathrm{~d} \bar{q} \mathrm{~d} q] \rightarrow \int \prod_{x \in \Lambda}\left[\mathrm{d} \bar{q}(x) \mathrm{d} q(x) \prod_{\mu} \mathrm{d} U_{\mu}(x)\right],
$$

where $\mathrm{d} U$ is the $\mathrm{SU}(3)$ Haar measure. If the lattice has a finite number of points the resulting functional integral is well defined without the necessity of fixing a gauge, and we have a solid non-perturbative definition of the theory.

One simple feature of the QCD action is that it is bilinear in the quark fields, and so we can "integrate" them exactly using the rules of Grassmannian integration. An expectation value then reduces to

$$
\langle\mathcal{O}[U, \bar{q}, q]\rangle \propto \int[\mathrm{d} U] \exp (-S[U]) \mathcal{O}[U, D[U]]
$$

where the action above includes the contribution from the internal quark loops which is encoded in the determinant of the Dirac operator:

$$
S[U]=S_{\text {gauge }}[U]-\ln \operatorname{det}(i \gamma D[U]+m) .
$$

The problem thus reduces to the evaluation of an enormous integral over the lattice gauge fields only, which is done by sophisticated Monte Carlo methods which we shall not describe here. There is a huge saving in computation if one neglects the fermion determinant in (6). This procedure called the "Quenched approximation", which has been widely employed in the past in order to make the simulations feasible (and also to gain valuable experience in numerical methods), is completely ad hoc and hence introduces uncontrolled systematic errors. Serious unquenched simulations (keeping the fermion determinant) have only been performed in recent years.

\subsection{Systematic errors}

Although the starting point of a lattice computation is completely well defined there are unfortunately various sources of systematic errors in obtaining physical quantities. The three main ones are: 1) finite ultraviolet cutoff (finite lattice spacing): here the effects are assumed to be dominantly power-like in $a$ and quantitatively described by Symanzik's effective langrangian [2, 3]. 2) Since we work with a finite number of points the volume is also finite. For large volumes the nature of the effects on various quantities have been analyzed by Lüscher [4]. 3) Finally, since the computational time depends quite strongly on the quark mass (see below), we are presently still working with quark masses larger than the accepted physical values (corresponding now to pion masses $m_{\pi}>\sim 300 \mathrm{MeV}$ ), and here the extrapolations are made using Chiral Perturbation Theory $(\chi \mathrm{PT})[5]$.

Many lattice projects invest a big effort in reducing and controlling these effects. In particular this involves establishing at which scales the theoretically expected behaviors set in for various observables. This activity is of utmost importance because it is our goal to obtain results from first principles with a sufficient accuracy, so that any deviation of theory from experiment could not be simply explained by saying that some systematic errors have been underestimated. 
When attempting to assess results of a lattice simulation for phenomenological applications, one should check whether and how the authors have taken into account the systematic errors mentioned above. At this point let me also draw attention to the fact that since lattice gauge theory computations only produce dimensionless numbers, only values for ratios of physical dimensionful quantities are determined. To quote numbers in $\mathrm{MeV}$ for masses (or decay constants etc) some scales have to be fixed by identifying some chosen subset with their experimental values. This is obviously a rather ad hoc procedure if one is not working with the physical number of degrees of freedom (e.g. in the quenched approximation or with a small number of flavors $N_{\mathrm{f}}=2$ ). The subsets chosen sometimes differ from one collaboration to another which often explains the apparent discrepancy in their quoted results.

\subsection{Lattice actions}

The plausible but yet unproven principle of universality leads to a great freedom in choosing an admissible QCD lattice action. Many different gauge and fermion actions, all maintaining locality, are presently in use by various collaborations. This rather confusing state of affairs has its advantages and disadvantages. One disadvantage is that raw data are often not cross-checked. On the other hand obtaining the same physical result (i.e. in the continuum limit) by various independent methods increases its credibility.

The fermion actions employed differ mainly on how chiral symmetry is treated. The original Wilson action [6], or its $\mathrm{O}(a)$ improved version [7], explicitly breaks chiral symmetry for zero bare quark mass, and the bare quark mass has to be tuned to regain the chiral Ward identities. It has the advantage of being conceptually simple. Staggered or Kogut-Susskind fermions [8], which break flavor symmetry at finite cutoff but have too much symmetry flavor symmetry in the continuum limit (see Subsection. 1.3), have the advantage that they are relatively cheap to simulate. Neuberger's overlap fermions [9], for which the Dirac operator obeys the Ginsparg-Wilson [10, 12], relation has an exact chiral symmetry [13] a, but they are very expensive. Kaplan's domain wall quarks [15] have an approximate chiral symmetry, and so too the implementation of the fixed-point action by Hasenfratz and Niedermayer $[11,12]$. Finally twisted mass QCD $[16,17]$ and chirally improved quarks $[18,19]$ are sorts of a compromises.

\subsection{Fourth root trick}

There have recently been strong claims of lattice phenomenological successes using staggered quarks $[20,21]$. But this formulation has problems when $N_{\mathrm{f}} / 4 \neq$ integer and to treat nondegenerate quarks (e.g. the strange quark) the so-called "rooting trick" was employed in which $\operatorname{Det}(i D+m)$ is replaced by its fourth $\operatorname{root} \operatorname{Det}(i D+m)^{1 / 4}$ by hand! The validity of such a manipulation is at first sight highly suspect since one would expect that it would cause violations of unitarity and locality. One outspoken opponent to its use is Creutz [22], but his critiques are not yet sufficiently strong, and indeed no one has yet been able to find a proof that the formulation is actually wrong. The topic is hotly debated in lattice workshops, as it will be again this year. The verdict of Sharpe in his plenary talk last year [23] with the catchy title "Rooted staggered fermions, good, bad or ugly?", was that

\footnotetext{
${ }^{a}$ differing from the continuum transformations by lattice artifacts and hence overcoming the NinomiyaNielsen theorem [14]
} 
they were "at least ugly". In any case it is important to appreciate that with our present level of understanding the formulation may be wrong, and hence any hence results obtained using it ${ }^{b}$ !

\subsection{Sofware advances; "breaking down the Berlin wall"}

A very good news in the past year was that Wilson quarks are not as expensive as previously thought, thereby eliminating the motivation of working with staggered quarks and invoking uncertain procedures. At the lattice workshop in Berlin in 2001 Ukawa [25] proposed the following formula for dependence of the number of operations in Teraflop years required to produce an ensemble of (statistically independent) field configurations $N$ on the spatial extent $L$, the lattice spacing $a$ and quark mass $m$ :

$$
N_{\text {Ukawa }}=5.00\left[\frac{20 \mathrm{MeV}}{m}\right]^{3}\left[\frac{0.1 \mathrm{fm}}{a}\right]^{7}\left[\frac{L}{3 \mathrm{fm}}\right]^{5} .
$$

(7) Figure 1: The cost formulae (7) (black) and

This at the time discouraging formula ${ }^{\mathrm{c}}$ (8) (red) versus $m$ for the case $a \simeq 0.08 \mathrm{fm}$ was not obtained by rigorous arguments but and $L=32 a \simeq 2.5 \mathrm{fm}$.

based on the experience gained by the $\mathrm{CP}$ -

PACS and JLQCD simulations. At last

year's lattice workshop Giusti [24] presented the following estimate for the same physical parameters but now based on the simulations of Ref. [26]:

$$
N_{\text {Giusti }}=0.05\left[\frac{20 \mathrm{MeV}}{m}\right]^{1}\left[\frac{0.1 \mathrm{fm}}{a}\right]^{6}\left[\frac{L}{3 \mathrm{fm}}\right]^{5} .
$$

What is striking is the reduction of the amplitude by a factor 100 , the significant reduction on the dependence on the quark mass, and also a reduction on the dependence on the lattice spacing! The enormous gain is more easily appreciated by inspecting Fig. 1. Similar performances to (8) were reported by other groups using various simulation techniques e.g. $[27,28]$. The impressive acceleration is mainly due to the progress in algorithms [28]-[31] and the inclusion of features to improve program efficiency (see e.g. [32]).

\subsection{Hardware}

The huge acceleration gained by the software has also been supplemented by the availability of powerful computer resources. Table 1 gives an incomplete list of the facilities used by the lattice community. It is to be noted that the peak performance has

\footnotetext{
$\mathrm{b}$ (unless one could argue that the wrong procedure effects the physical quantities under consideration only weakly)

${ }^{c}$ (given here explicitly for $N_{\mathrm{f}}=2$ flavors of $\mathrm{O}(a)$-improved Wilson quarks in a volume $V=2 L \times L^{3}$ and with degenerate quarks of mass $m=m_{\overline{\mathrm{MS}}}(2 \mathrm{GeV})$ )
} 
been entered; typical programs perform at $\sim 50 \%$ of the peak ${ }^{\mathrm{d}}$. Finally the upcoming International Lattice Data Grid [33] organizing the storing of gauge configurations generated in the time consuming dynamical simulations, will be a big asset which will aid the extraction of physical observables and further promote international cooperation.

\section{Observables}

In lattice simulations one measures Euclidean lattice correlation functions of the type $\left\langle\mathcal{O}_{1} \mathcal{O}_{2} \ldots\right\rangle$ where $\mathcal{O}_{1}$ are gauge invariant observables. For example $\langle\mathcal{O}(0) \mathcal{P}(t)\rangle$ for large $t$ gives information on the matrix element $\langle 0|\mathcal{O}(0)| P\rangle$ where $|P\rangle$ is the lowest state with quantum numbers of the local operator $\mathcal{P}$. One is usually interested in the connected parts of such correlation functions and thus the computation tends to become very difficult if the number of operators in the product is large because of severe statistical fluctuations.

Nevertheless one can obtain information on many quantities of phenomenological relevance to hadron structure including spectra, resonance properties and phase shifts, hadronic contributions to $g-2$, running couplings, meson distribution amplitudes, elastic and transition form factors and moments of (generalized) structure functions. Because of lack of space I will in this section restrict myself to the last three topics, and finally in Sect. 3 briefly report on running couplings. For a thorough review of lattice results on hadron structure I recommend the article by Orginos [34].

\subsection{Meson distribution amplitudes}

Meson distribution amplitudes are defined (in Minkowski space) by matrix elements of bilocal operators e.g. the kaon distribution amplitude $\phi_{K}(\xi, \mu)$ is given by:

\begin{tabular}{|l|l|c|}
\hline Type & Facility & Peak \\
\hline Custom & Blue GeneL, Jülich & 46 \\
computers & Blue GeneL, KEK & 57 \\
\hline PC clusters & Altix, LRZ & 26 \\
& PACS-CS, Tsukuba & 14 \\
\hline Self-built & QCDOC, BNL & 20 \\
& APE-next, Rome & 8 \\
\hline
\end{tabular}

Table 1: Computing facilities, with peak performance in TFlops.

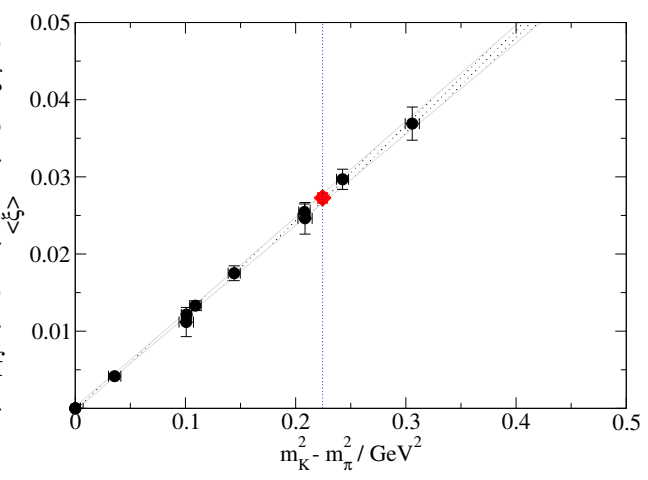

Figure 2: QCDSF [37] $N_{\mathrm{f}}=2$ results for the first moment of the kaon distribution function.

$$
f_{K} i p_{\rho} \int_{-1}^{1} \mathrm{~d} \xi \mathrm{e}^{i \xi p \cdot z} \phi_{K}(\xi, \mu)=\left\langle 0\left|\bar{q}(z) \gamma_{\rho} \gamma_{5} P \exp \left[i \int_{-z}^{z} A(x) \cdot \mathrm{d} x\right] s(-z)\right| K(p)\right\rangle_{z^{2}=0} .
$$

${ }^{\mathrm{d}}$ Note also that some of the facilities are shared with groups working on other topics. 
The distribution is not directly measurable on the lattice however its (low) moments

$$
\left\langle\xi^{n}\right\rangle_{K}(\mu)=\int_{-1}^{1} \mathrm{~d} \xi \xi^{n} \phi_{K}(\xi, \mu)
$$

are, since they are expressed as matrix elements of local operators:

$$
\langle\xi\rangle_{K}(\mu) f_{K} p_{\rho} p_{\nu}=\left\langle 0\left|\bar{q}(0) \gamma_{\rho} \gamma_{5} \stackrel{\leftrightarrow}{D}_{\nu} s(0)\right| K(p)\right\rangle .
$$

These were one of the first quantities to be measured in (quenched) lattice simulations [35, 36]. Fig. 2 shows recent measurements by QCDSF [37] with $N_{\mathrm{f}}=2$ of the first moment $\langle\xi\rangle_{K}$ as a function of the meson (squared) mass difference. A nice feature is that it approaches zero in the degenerate case as expected. Interpolating to the physical value of $m_{K}^{2}-m_{\pi}^{2}$ yields $\langle\xi\rangle_{K}^{\overline{M S}}(2 \mathrm{GeV})=0.027(02)$. This is consistent with the central value $0.030(12)$ of the computation using QCD sum rules [38, 39, 40]; the quoted errors of the lattice computation are smaller but these do not include some systematic errors e.g. those due to lattice artifacts. Another computation [41] using (RBC/UKQCD) configurations from simulating $N_{\mathrm{f}}=2+$ 1 dynamical flavors of domain wall quarks, yields a similar number 0.032(03). QCDSF also quote a result for the second moment of the kaon $\mathrm{DF}\left\langle\xi^{2}\right\rangle_{K}^{\overline{\mathrm{MS}}}(2 \mathrm{GeV})=0.26(2)$, and furthermore for the corresponding value for the pion $\left\langle\xi^{2}\right\rangle_{\pi}^{\overline{\mathrm{MS}}}(2 \mathrm{GeV})=0.27(4)$ which turns out to be practically the same.

\section{$2.2 \quad$ Nucleon axial coupling}

The nucleon axial coupling has been computed by many groups [42]-[46] with encouragingly consistent results. These are summarized in Fig. 3 which also shows that dependence of $g_{A}$ on $m_{\pi}$ is surprisingly weak.

Note however that for this quantity there are rather significant finite volume effects which must be taken into account. At LAT06 Orginos [34] quoted a value $g_{A}\left(m_{\pi}=140 \mathrm{MeV}\right)=1.23(8)$ consistent with the experimental value of $1.2695(29)$ but much more inaccurate. In any case $_{\mathrm{g}_{\mathrm{A}}}$ to compare with experiment in more detail isospin breaking effects would have to be included. To enable a better comparison with $\chi \mathrm{PT}$ [47] measurements with $N_{\mathrm{f}}=2+1$ dynamical quarks at smaller $m_{\pi}$ are under way.

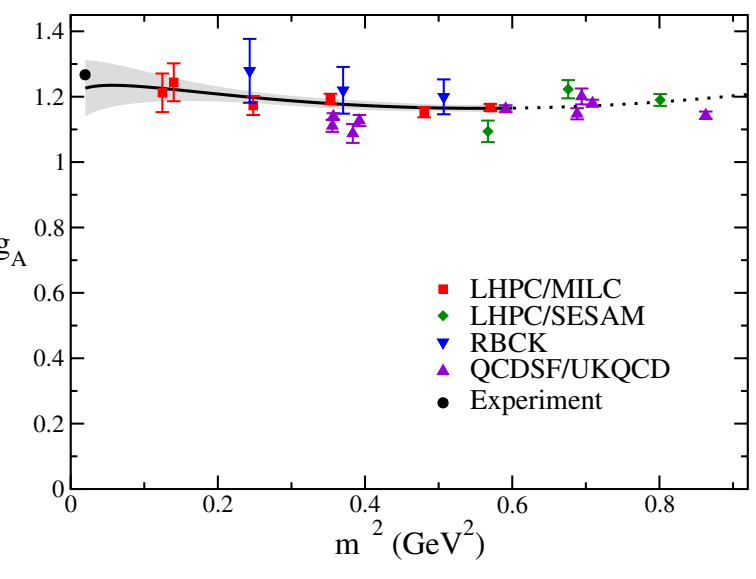

\subsection{Nucleon form factors}

Figure 4 shows some recent measurements Figure 3: Dependence of lattice values for $g_{A}$

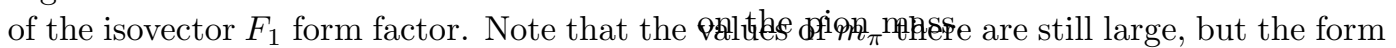
factor seems to approach the experimental measurements as $m_{\pi}$ decreases. 


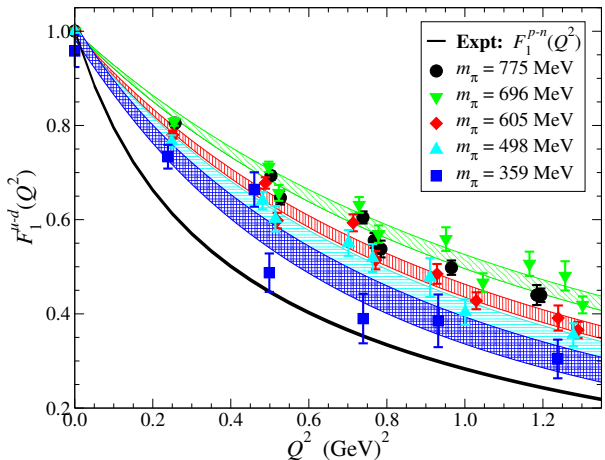

Figure 4: LHPC '06 measurement of the nucleon isovector $F_{1}$ form factor

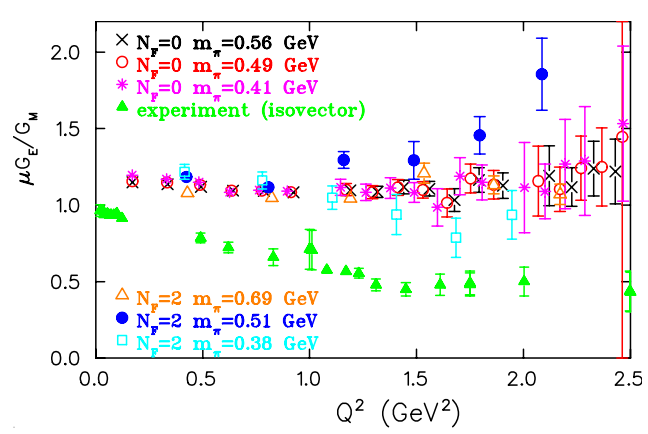

Figure 5: Ratio of electric and magnetic isovector form factors

One general point to appreciate is that if periodic boundary conditions (pbc) are used, then momentum components are quantized in units $2 \pi / L$ where $L$ is the spatial extent of the lattice. For example a lattice of size $L=24 a$ with $a=0.1 \mathrm{fm}$ gives $2 \pi / L \sim 0.52 \mathrm{GeV}$. Hence typically only quite widely separated points on the curves are obtained. In addition one has the problem that lattice artifacts become larger at the higher momenta. An idea to improve this situation proposed by Bedaque [48], and applied e.g. in [49], is to use twisted pbc where the allowed momentum coefficients have the form $p_{i}=\left(2 \pi n+\theta_{i}\right) / L$. This yields more points on the curves at the price that each different set of values for the twists $\theta_{i}$ requires a separate simulation.

An illustration that not everything is so rosy yet is Fig. 5 from Alexandrou et al [50] showing a measurement of the ratio of electric and magnetic isovector form factors. As one can see there is presently an apparent disagreement of this measurement with with that obtained by the preferred JLab polarization transfer experiments [51]. In fact the result resembles more the older experimental findings using the Rosenbluth separation technique [52]. But the authors of Ref. [50] caution that there may be strong lattice artifacts which still need to be examined.

\subsection{Moments of structure function}

Figure 6 shows some data for the average value for the isovector first moment $\langle x\rangle_{u-d}$. The squares are points from a quenched simulation (QCDSF) with the overlap operator, which are quite far from the accepted experimental value. The circles are data from the LHPC group [53] for $N_{\mathrm{f}}=2+1$ dynamical (staggered) quarks (at one lattice spacing $a \sim 0.125 \mathrm{fm}$ ). This data does not yet show the expected chiral logarithm [54]

$$
\langle x\rangle_{u-d}=C\left[1-r^{2}\left(A \ln r^{2}+B\right)+\ldots\right],
$$

where $r=m_{\pi} /\left(4 \pi f_{\pi}\right)$ with a computed large amplitude $A=6 g_{A}^{2}+2 \sim 11$. But if one extrapolates the present data using the formula above one indeed obtains a value consistent with experiment.

The situation for the ratios of moments is much the same; i.e. fitting to $\chi \mathrm{PT}$ one obtains good agreement with experiment for a wide range of moments as illustrated in Fig. 7. 


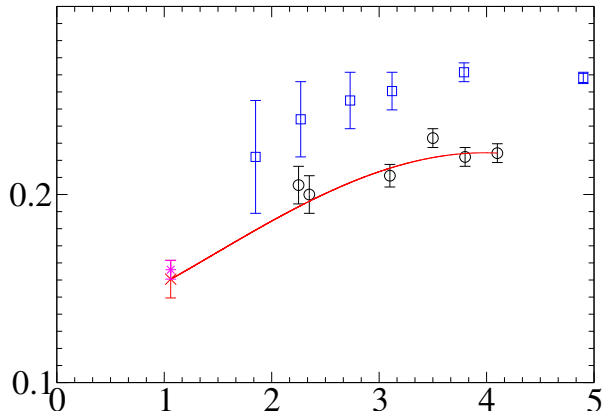

Figure 6: Isovector first moment $\langle x\rangle_{u-d}$. The curve is an extrapolation using $\chi \mathrm{PT}$.

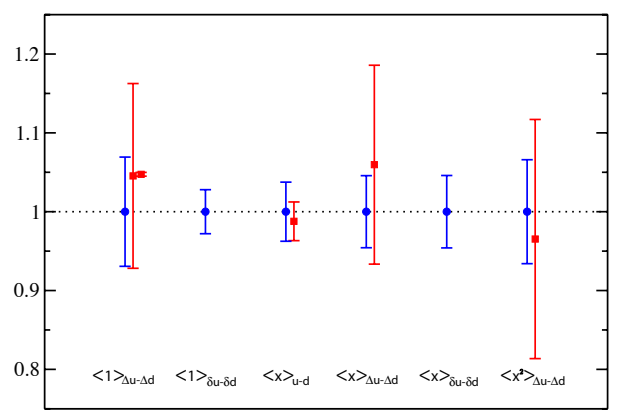

Figure 7: Ratios of Moments: Lattice/DIS

\section{Running couplings}

Various physical processes measured can be computed at high energies within the framework of perturbation theory. If the perturbative expression for a given process $P(s)$ at scale $s$ starts with $c \alpha^{n}$ (for some perturbative renormalized coupling $\alpha$ ) then we can define a nonperturbative running coupling by $\alpha[P](s)=[P(s) / c]^{1 / n}$. Such running couplings are often defined down to low energies where perturbation theory is surely no longer valid.

What is usually done is to match the measurement of $P(s)$ with its perturbative expression in order to obtain values of the coupling in the $\overline{\mathrm{MS}}$-scheme $\alpha_{\overline{\mathrm{MS}}}(s)$ in the energy range of the experiment. A collection of the values obtained from various experiments is then compared to the function $\alpha_{\overline{\mathrm{MS}}}(s)$ which is the solution of a renormalization group equation with the $\beta$-function computed to a certain order. If good agreement is observed it is often said that "running of the coupling has been observed". This is a mild misuse of words since we are patching together various experiments and also the function $\alpha_{\overline{\mathrm{MS}}}(s)$ certainly runs! We should rather just say we observe satisfactory agreement with the perturbative prediction (which entails asymptotic freedom).

To actually observe running of a given physical coupling we should measure it over a wide range of energies and check at which energy perturbative behavior seems to sets in (to a given accuracy). At that stage

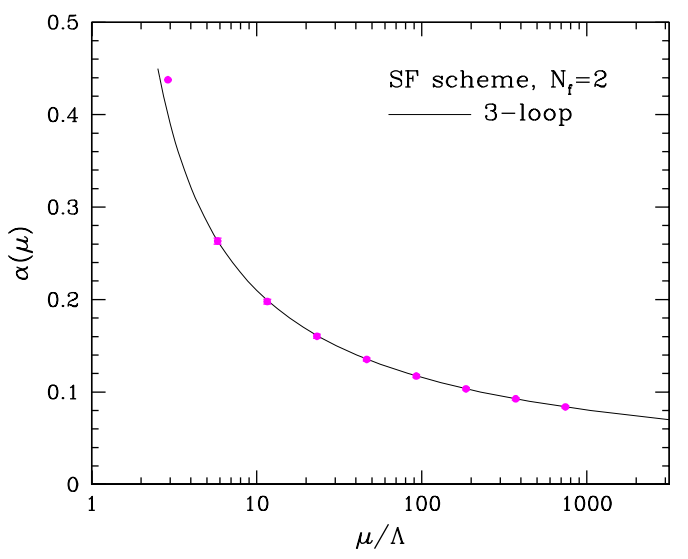
we can estimate the associated $\Lambda$-parameter and convert to the $\overline{\mathrm{MS}}$ scheme if desired.

Figure 8: Running coupling in the SF scheme for $N_{\mathrm{f}}=2$. 
There are unfortunately few experiments which cover a sufficiently wide energy range. Indeed some experiments cover only a short range at relatively low energies. In this case one has to resort to the mere hope that perturbative behavior has already set in. This can certainly lead to uncontrolled errors. In the lattice literature there are analogous procedures. An example is the much publicized work [20] who quote a value for $\alpha_{\overline{\mathrm{MS}}}\left(m_{Z}\right)$ with small error bars. In an admittedly over-simplified presentation, their result is basically obtained by measuring various quantities e.g. charmonium splittings at a given value of the bare coupling $g_{0}$, thereby extracting a value of the lattice spacing $a\left(g_{0}\right)$ in physical units. Subsequently an estimate of $\alpha \overline{\mathrm{MS}}\left(a\left(g_{0}\right)\right)$ is obtained in terms of a (modified) perturbative relation to $g_{0}$. Finally they use the RG equation to evolve from the charm scale up to the $\mathrm{Z}$ mass. No actual running is measured; moreover the non-perturbative measurements used the questionable fourth-root trick discussed in Subsect. 1.3. Its theoretical status should certainly be taken into account when making comparisons with experimental results, which albeit often also have their own sources of uncontrolled systematic errors $[55,56]$.

A more satisfactory job, in my opinion, is that what has been done by the Alpha Collaboration [57]. They indeed measure a special non-perturbatively defined running coupling ${ }^{\mathrm{e}}$ over a wide range of energies, including careful extrapolations to the continuum limit. They first check that perturbation theory indeed seems to set in, as seen in Fig. 8. Amusingly this coupling runs according to 3-loop PT right down to relatively low energies, but this must certainly not be misunderstood as a universal property of running couplings. Unfortunately their computations are so far only for the unphysical case of $N_{\mathrm{f}}=2$ flavors. Converting to the $\overline{\mathrm{MS}}$ scheme at their highest measured energy they obtain the result $\left[\Lambda_{\overline{\mathrm{MS}}} r_{0}\right]^{(2)}=0.62(6)$; if one sets the Sommer scale [58] to its phenomenological value $r_{0}=0.5 \mathrm{fm}$ one obtains $\Lambda_{\overline{\mathrm{MS}}}^{(2)}=245(23) \mathrm{MeV}$, but as discussed in Subsect. 1.1 the choice of scale introduces an additional systematic error if one wishes to compare with experiment.

In Fig. 9 they plot the extracted nonperturbative beta function for $N_{\mathrm{f}}=0,2$. One first clearly sees the expected contribution from the fermions. Furthermore one observes the onset of a deviation from perturbative behavior at the lowest energies.

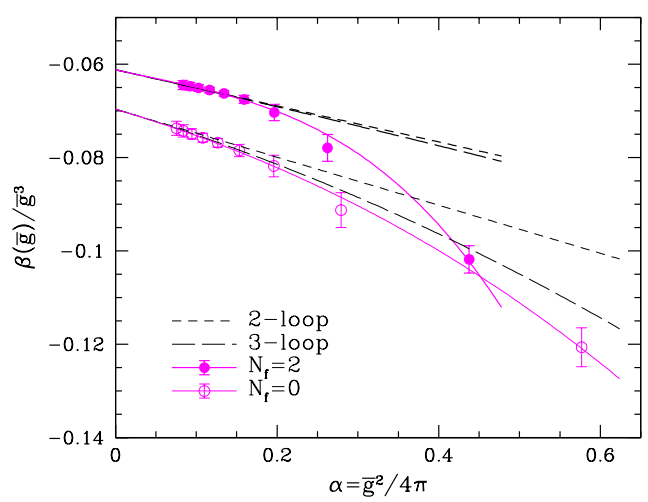

Figure 9: Comparison of non-perturbative to perturbative $\beta$-functions.

\section{Summary}

Much algorithmic progress in lattice QCD has been achieved in the last 5 years. Serious dynamical quark simulations of QCD with larger physical volumes and relatively small

${ }^{e}$ which depends on the physical volume, and defined through the Schroedinger functional 
lattice spacings are now under way. However a big effort is still needed to simulate with the physical value of the pion $m_{\pi} \sim 140 \mathrm{MeV}$. Chiral logarithms have not yet been clearly seen, but lattice simulations combined with extrapolations using $\chi \mathrm{PT}$ gives reasonable agreement with experiment for many observables describing hadronic structure. Finally there is a huge effort to control the various systematic errors which is as essential for the quality of a lattice computation as it is for any real experiment.

\section{Acknowledgments}

I would like to thank Kostas Orginos for sending me the figures which appeared in his lattice proceedings, some of which are reproduced here. I also thank Meinulf Goeckeler for his suggestions concerning the selection of topics covered in Sect. 2.

\section{References}

[1] Slides: http: //indico. cern. ch/contributionDisplay . py? contribId=8\&sessionId=2\&conf Id=9499

[2] K. Symanzik, Nucl. Phys. B226 187 (1983).

[3] M. Lüscher, In Les Houches 1984 Proceedings, Critical Phenomena, Random Systems, Gauge Theories, 359.

[4] M. Lüscher, Commun. Math. Phys. 104177 (1986); ibid 105153 (1986); Nucl. Phys. B354 531 (1991); ibid B364 237 (1991).

[5] J. Gasser, H. Leutwyler, Ann. Phys. 158142 (1984).

[6] K. Wilson, Phys. Rev. D10 2445 (1974).

[7] B. Sheikholeslami, R. Wohlert, Nucl. Phys. B259 572 (1985).

[8] L .Susskind, Phys. Rev. D16 3031 (1977).

[9] H. Neuberger, Phys. Lett. B427 353 (1998);ibid B414 341 (1998).

[10] P.H. Ginsparg, K.G. Wilson, Phys. Rev. D25 2649 (1982).

[11] P. Hasenfratz, Nucl. Phys. Proc. Suppl. 6353 (1998).

[12] F. Niedermayer, Nucl. Phys. Proc. Suppl. 73105 (1999).

[13] M. Lüscher, Phys. Lett. B428 342 (1998).

[14] N. Nielsen,M. Nimomiya, Phys. Lett. B105 219 (1981).

[15] D. Kaplan, Phys. Lett. B288 342 (1992).

[16] R. Frezzotti, P.A. Grassi, S. Sint, P. Weisz, JHEP 0108:058 (2001).

[17] R. Frezzotti, G.C, Rossi, JHEP 0408:007 (2004).

[18] C. Gattringer, Phys. Rev. D63 114501 (2001).

[19] C.B. Lang, P. Majumdar, W. Ortner, Phys. Rev. D73 034507 (2006).

[20] Q. Mason et al. (HPQCD), Phys. Rev. Lett. 95052002 (2005).

[21] C.T.H. Davies et al. Phys. Rev. Lett. 92022001 (2004);Q. Mason et al. (HPQCD), Phys. Rev. D73 114501 (2006).

[22] M. Creutz, hep-lat/0701018v2.

[23] S. Sharpe, PoS LAT2006:022 (2006).

[24] L. Giusti, PoS LAT2006:009 (2006).

[25] A. Ukawa et al. (CPPACS and JLQCD), Nucl. Phys. Proc. Suppl. 106195 (2002).

[26] L. Del Debbio, L. Giusti, M. Lüscher, R. Petronzio, N. Tantalo, JHEP 0702:056 (2007); JHEP 0702:082 (2007).

[27] M.A Clark, A.D. Kennedy, Phys. Rev. Lett. 98051601 (2007).

[28] C. Urbach, K. Jansen, A. Shindler, U. Wenger, Comput. Phys. Commun. 17487 (2006).

[29] M. Hasenbusch, Phys. Lett. B519 177 (2001).

[30] M. Lüscher, JHEP 0305:052 (2003). 
[31] M.A Clark, A.D. Kennedy, Nucl. Phys. Proc. Suppl. 129850 (2004).

[32] M. Lüscher, Nucl. Phys. Proc. Suppl. 10621 (2002).

[33] K. Jansen, hep-lat 0609012.

[34] K. Orginos, Pos (LATT2006) 018.

[35] A.S. kronfeld, A.M. Photiadis, Phys. Rev. D31 2939 (1985).

[36] G. Martinelli, C.T. Sachrajda, Phys. Lett. B190 152 (1987).

[37] V.M. Braun et al., Phys. Rev. D74 074501 (2006).

[38] A. Khodjamirian, T. Mannel, M. Melcher, Phys. Rev. D70 094002 (2004).

[39] V.M. Braun, A. Lenz, Phys. Rev. D70 074020 (2004).

[40] P. Ball, R. Zwicky, Phys. Lett. B633 289 (2006); JHEP 02034 (2006).

[41] P.A. Boyle et al., Phys. Lett. B641 67 (2006).

[42] S.R. Beane, M.J.Savage, Phys. Rev. D70 074029 (2004).

[43] S. Ohta, K. Orginos (RBCK), Nucl. Phys. Proc. Suppl. 140396 (2005).

[44] H.W. Lin, S. Ohta, hep-lat/0610028.

[45] A. Ali Khan et al. (HPQCD and UKQCD), Nucl. Phys. Proc. Suppl. 153128 (2006).

[46] D. Dolgov et al., Phys. Rev. D66 034506 (2006).

[47] V. Bernard, U.G. Meissner, Phys. Lett. B639 278 (2006).

[48] P.F. Bedaque, Phys. Lett. B593 82 (2004).

[49] J.M. Flynn, A. Juttner, C.T. Sachrajda [UKQCD], Phys. Lett. B632 313 (2006).

[50] C. Alexandrou, G. Koutsou, J.W. Negele, A. Tsapalis, Phys. Rev. D74 034508 (2006).

[51] V. Punjabi et al., Phys. Rev. C71 055202 (2005).

[52] L. Andivahis et al., Phys. Rev. D50 5491 (1994);

M.E. Christy et al. (E94110), Phys. Rev. C70 015206 (2004).

[53] R.G. Edwards et al. (LHPC), Proc. Sci. LAT2006 (2006).

[54] D. Arndt, M.J. Savage, Nucl. Phys. A697 429 (2002).

[55] S. Bethke, Prog. Part. Nucl. Phys. 58351 (2007).

[56] S. Kluth, hep-ex/0609010.

[57] M. Della Morte et al. [ALPHA Collab.], Nucl. Phys. B713 378 (2005).

[58] R. Sommer, Nucl. Phys. B411 839 (1994). 